\title{
Demographic and socio-economic predictors of diet quality among adults in Bosnia and Herzegovina
}

\author{
Selma Gicevic ${ }^{1, *}$ (1), Audrey J Gaskins 1,2, Teresa T Fung ${ }^{1,3}$, Bernard Rosner ${ }^{2,4}$, \\ Edin Sabanovic ${ }^{5}$, Jelena Milesevic ${ }^{6}$, Agnes Kadvan ${ }^{6}$, Emir Kremic ${ }^{7}$ and \\ Walter Willett ${ }^{1,2,8}$
}

'Department of Nutrition, Harvard T.H. Chan School of Public Health, 655 Huntington Avenue, Boston, MA 02115 , USA: ${ }^{2}$ Channing Division of Network Medicine, Department of Medicine, Brigham and Women's Hospital, Boston, MA, USA: ${ }^{3}$ Department of Nutrition, Simmons University, Boston, MA, USA: ${ }^{4}$ Department of Biostatistics, Harvard T.H. Chan School of Public Health, Boston, MA, USA: ${ }^{5}$ Agency for Statistics of Bosnia and Herzegovina, Sarajevo, Bosnia and Herzegovina: ${ }^{6}$ Centre of Research Excellence in Nutrition and Metabolism, Belgrade, Serbia: ${ }^{7}$ Institute for Statistics of the Federation of Bosnia and Herzegovina, Sarajevo, Bosnia and Herzegovina: ${ }^{8}$ Department of Epidemiology, Harvard T.H. Chan School of Public Health, Boston, USA

Submitted 16 November 2018: Final revision received 29 March 2019: Accepted 26 April 2019: First published online 9 August 2019

\begin{abstract}
Objective: To evaluate associations of demographic and socio-economic factors with diet quality among population subgroups in Bosnia and Herzegovina (B\&H).

Design: A cross-sectional analysis of 2017 B\&H dietary survey data. Diet quality was assessed by the Prime Diet Quality Score (PDQS) utilizing data from two non-consecutive $24 \mathrm{~h}$ diet recalls. Socio-economic variables were extracted from the 2015 B\&H Household Budget Survey. Homogeneity of means across population subgroups was evaluated using multivariable regression.

Setting: B\&H population survey.

Participants: A population-based sample of 853 adults.

Results: The mean PDQS was $15 \cdot 8$ (range 7-28 out of a possible 42 points). In general, Bosnian adults had low PDQS due to high intakes of refined grains, high-fat dairy and processed meats, and low intakes of whole grains, nuts and fish. The PDQS was significantly higher $(P<0 \cdot 0001)$ among older individuals (17.0) compared with those in the youngest group (14.5), among individuals living in the central and northern regions (16.5) compared with those living in the south $(15 \cdot 1 ; P<0 \cdot 0001)$, and among people who are married/cohabitating (16.1) $v$. single $(14 \cdot 8 ; P=0 \cdot 02)$. In energy-adjusted models, socio-economic status $(P=0.04)$ and tertiles of household spending $(P=0.002)$ were inversely associated with the PDQS.

Conclusions: Diet quality in this population was low. Young and middle-aged individuals, singles and those living in the south had significantly lower quality diets compared with other subgroups. Public health action is needed to promote higher consumption of whole grains, nuts and fish, and a higher variety of fruits and vegetables.
\end{abstract}

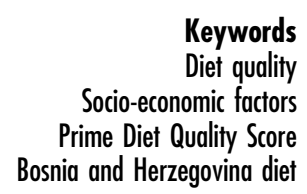

Keywords Diet quality Prime Diet Quality Score Bosnia and Herzegovina diet
Dietary surveys represent an important source of data on population dietary behaviours associated with health and disease, and are a prerequisite for the creation and tracking of nutrition-related public health policies and programmes. While mean nutrient and food intakes on a population level are valuable, they can often mask inadequate or excess intakes in specific demographic or socio-economic subgroups that may require additional attention. The literature from high-income countries ${ }^{(1-3)}$ has shown significant and widening differences in diet quality by socio-economic status (SES), with disadvantaged socio-economic groups consuming more affordable, nutrient-poor foods compared with high-SES groups. The nutrition transition from traditional diets to more Western-style diets has been well 
documented in many low- and middle-income countries of Asia, Africa and Latin America ${ }^{(4-7)}$. These eating patterns are characterized by increased intakes of energy, meat, SFA, sugar and refined grains coupled with reduced intakes of fibre, fruits and vegetables. While the positive gradient in diet quality by SES is clear in high-income countries, some low- and middle-income countries undergoing nutrition transition have experienced a different pattern ${ }^{(5,6)}$. Specifically in these low- and middle-income countries, urban and high-income groups seem to more readily embrace unhealthy Western-style diets, while low-income and rural groups tend to preserve traditional diet features over a longer period of time by consuming more vegetables, oils and whole grains, and less meat and SFA.

Bosnia and Herzegovina (B\&H) was a part of the socialist Yugoslavia until its breakdown in 1992, followed by an armed conflict in 1992-1995. The country is organized into two administrative units, the Federation of Bosnia and Herzegovina (FB\&H) and the Republika Srpska. B\&H's economy underwent transition to a market economy in the late 1990s, but is still catching up with more developed neighbouring countries ${ }^{(8,9)}$. Located in the Balkan Peninsula, its northern parts are characterized by mountain ranges and a moderate continental climate, while southern parts bordering with the Adriatic have a significantly warmer, Mediterranean climate. The Bosnian and Adriatic fishery is a great source of both wild and farmed fish ${ }^{(10,11)}$, while the diverse terrain is suitable for growing an assortment of fruits and vegetables, nuts and other crops. Among the locally available foods rich in key nutrients and affordable are trout, sardines, mackerel, walnuts, rye, buckwheat, carrots and collard greens.

Health statistics indicate that $\mathrm{B} \& \mathrm{H}$ is at an advanced stage of epidemiological transition with CVD as the leading cause of mortality ${ }^{(12,13)}$ and type 2 diabetes and diet-related cancers on the rise ${ }^{(12,13)}$. Overweight and obesity affect over half of the country's population ${ }^{(12)}$. While the literature on pre-war and post-war dietary habits of former Yugoslavian and Bosnian populations is sparse, the Seven Countries Studies ${ }^{(14,15)}$ identified geographic variations in the type of fat consumed among men in pre-war Yugoslavia, while a study among Bosnian immigrants in Sweden ${ }^{(16)}$ and the 2012 B\&H Health Survey ${ }^{(13)}$ suggested high intakes of refined wheat flour and sugary products (such as white bread, pies and sweet pastry) in Bosnian diets. We were, however, unable to find literature on Bosnian or West Balkans changes in dietary habits over the past decades, nor data on demographic (e.g. sex, age, marital status) or socio-economic (e.g. education, wealth, place of residence) differences in diet within the country. It is therefore unclear whether nutrition transition in this region has followed the patterns identified in other low- and middle-income countries. To address this gap, we evaluated the demographic and socio-economic predictors of nutrient and food intakes, as well as of the overall diet quality, among adults in B\&H.
Table 1 Characteristics of participants in the Bosnia and Herzegovina dietary survey (2017)

\begin{tabular}{|c|c|c|c|c|}
\hline & \multicolumn{2}{|c|}{$\begin{array}{l}\text { Male } \\
(n 371)\end{array}$} & \multicolumn{2}{|c|}{$\begin{array}{l}\text { Female } \\
(n 482)\end{array}$} \\
\hline & $n$ & $\%$ & $n$ & $\%$ \\
\hline \multicolumn{5}{|l|}{ Age group } \\
\hline $18-40$ years & 111 & 30 & 130 & 27 \\
\hline $41-60$ years & 157 & 42 & 227 & 47 \\
\hline$\geq 61$ years & 103 & 28 & 125 & 26 \\
\hline \multicolumn{5}{|l|}{ Place of residence } \\
\hline Urban & 167 & 45 & 206 & 43 \\
\hline \multicolumn{5}{|l|}{ Education level (study participant) } \\
\hline$<12$ years (less than high school) & 53 & 14 & 213 & 44 \\
\hline 12 years (high school diploma) & 267 & 72 & 216 & 45 \\
\hline $\begin{array}{l}>12 \text { years (some higher education/higher } \\
\text { education diploma) }\end{array}$ & 51 & 14 & 53 & 11 \\
\hline \multicolumn{5}{|l|}{ Socio-economic status (SES) } \\
\hline Low SES & 121 & 32 & 172 & 36 \\
\hline Medium SES & 218 & 59 & 280 & 58 \\
\hline High SES & 32 & 9 & 30 & 6 \\
\hline \multicolumn{5}{|l|}{ Geographic location } \\
\hline Bosnia (north) & 238 & 64 & 348 & 72 \\
\hline Herzegovina (south) & 132 & 36 & 134 & 28 \\
\hline \multicolumn{5}{|l|}{ BMI $\left(\mathrm{kg} / \mathrm{m}^{2}\right)$} \\
\hline$<25 \cdot 0$ & 98 & 27 & 163 & 34 \\
\hline $25 \cdot 0-29 \cdot 9$ & 190 & 51 & 175 & 36 \\
\hline$\geq 30 \cdot 0$ & 83 & 22 & 144 & 30 \\
\hline Chronic disease & 113 & 30 & 195 & 40 \\
\hline Diabetes & 19 & 5 & 38 & 8 \\
\hline CVD & 66 & 18 & 122 & 25 \\
\hline \multicolumn{5}{|l|}{ Marital status } \\
\hline Single & 61 & 17 & 55 & 11 \\
\hline Married/living with partner & 291 & 78 & 329 & 68 \\
\hline Divorced/widowed & 19 & 5 & 98 & 20 \\
\hline
\end{tabular}

\section{Methods}

\section{Study design and population}

We used data from the 2017 B\&H dietary survey that included a population-based sample of 872 persons aged 18 years or over residing in FB\&H. This was a cluster randomized sub-sample of households participating in the 2015 Household Budget Survey (HBS 2015) ${ }^{(17)}$ in B\&H, used to minimize sampling costs and to allow linking of dietary and socio-economic data. HBS is a nationally representative, stratified random sample of households. The non-response rate of $34 \%$ in our study was partially due to an outdated sampling frame and frequent in-country migrations, and is similar to other dietary surveys ${ }^{(18)}$. Nineteen persons were excluded due to systematic interviewer error, resulting in the final sample of 853 persons (482 women and 371 men; Table 1). Dietary data were collected by trained interviewers in the form of two non-consecutive $24 \mathrm{~h}$ recalls (the first one in person, the second one over the telephone 4-30 d later) uniformly across four seasons with the weekday to weekend ratio of 7:3. Data were subsequently linked to socio-economic data from the HBS 2015. Weight and height data were self-reported; participants were, however, given a chance to use an interviewer-provided portable scale, measuring 
tape and written instructions to take these measures during interview if they were unsure.

\section{Demographic and socio-economic variables}

We used the Organisation for Economic Co-operation and Development-modified equivalence scale ${ }^{(19)}$ to standardize household spending levels by household size and then classified this variable into tertiles (low, medium, high). Education level of the head of household was categorized as follows: less than high school ( $<12$ years), high school completed (12 years) and some higher education or higher education diploma ( $>12$ years). A composite socioeconomic (SES) score (Table 1) was created to combine the effect of education and wealth. Participants were categorized into the 'high SES' category if the household head had $>12$ years of education and the household spending was in the highest tertile; into 'low SES' if the household head's education was $<12$ years and the spending was in the lowest tertile; everyone else was categorized as 'medium SES'. We also stratified participants by the place of residence using the official national statistics agency classification into 'urban', defined as residing in settlements with a 'city' status, and 'non-urban', that included semi-urban and rural settlements. Further, participants were classified by geographic location into those residing in the 'north' (municipalities in Bosnia, northern and central regions) or 'south' (municipalities in Herzegovina, southern regions). We also classified our participants by age group (18-40, 41-60 and $\geq 61$ years), BMI $\left(<25 \cdot 0,25 \cdot 0-29 \cdot 9\right.$ and $\left.\geq 30 \cdot 0 \mathrm{~kg} / \mathrm{m}^{2}\right)$ and marital status (single, married/cohabitating and divorced/widowed).

\section{Dietary assessment}

To determine intake quantities during interviews, we utilized the DIET-ASSESS Food Atlas ${ }^{(20,21)}$ which uses colour images of eating utensils, dishes, foods and meals to aid in the estimation of portion sizes during the in-home interviews. A short version of this food atlas was left with respondents to be used during telephone interviews. Nutrient intakes were calculated by DIET ASSESS \& PLAN advanced dietary assessment software ${ }^{(22)}$ linked to the Balkans food composition database. We focused on the key nutrients associated with health and disease, and further restricted our analysis to nutrients with confirmed validity in the Balkans food composition database. Data on trans-fatty acids and free/added sugars were not available in the database; hence we excluded these nutrients from our analyses. Individual foods were categorized into food groups of interest. 'Fruit' and 'vegetables' categories included only whole fruits and vegetables, while juices and spreads were categorized separately. Low-fat dairy included products with $2 \%$ milk fat or less. Ingredients of composite dishes were incorporated into several categories as appropriate (e.g. sugar from a cake with nuts would be added to the 'sweets' category while nuts would be counted as 'nuts'). All values were calculated as grams or millilitres per day and included non-consumers.

\section{Prime Diet Quality Score}

The Prime Diet Quality Score (PDQS) ${ }^{(23,24)}$ is a food-based, twenty-one-component, dietary score consisting of twelve 'adequacy', seven 'moderation' and two populationspecific components (for adults, only low-fat dairy foods are coded as an adequacy component, while high-fat dairy and eggs are not coded). The score was developed and successfully evaluated by comparison with a comprehensive FFQ in a high-income country setting ${ }^{(23,24)}$. In two studies of US women, higher score on the PDQS was found to predict lower risk of $\mathrm{CHD}^{(23)}$ and gestational diabetes mellitus $^{(24)}$. We adapted the scoring criteria for use with the two days of $24 \mathrm{~h}$ recall data. Specifically, for each 'adequacy' component, participants were assigned a ' 0 ' if no foods from a component were consumed in at least a minimum quantity during both days, a ' 1 ' if foods from a component were consumed in an amount above the minimum cut-off point on one day out of the two, and ' 2 ' if at least one food from a food component was consumed at or above minimum amount on both days. The coding was reversed for the 'moderation' components. The number of occasions a person consumed foods during a day from a component were not taken into account, i.e. any frequency above 1 was coded as ' 1 '. In order to preserve the original scoring of up to 42 points (representing a high-quality diet), we assigned 2 points to all participants for the 'eggs' component, keeping it as a neutral component. We set 'minimum amount per meal' cut-off points for each component based on expert knowledge, specific food portion sizes and distributions of actual intakes in the 2017 Bosnia diet survey ${ }^{(25,26)}$. The online supplementary material, Supplemental Table S1, gives details about foods included in each component and the cut-off points per meal.

\section{Statistical analysis}

We calculated nutrient and food intakes as a simple average of two days for each participant while the total PDQS was based on intakes from both days. Geometric means and SE were calculated from log-transformed nutrients. Gamma distribution of food groups was assumed to accommodate their skewed distributions. The PDQS was normally distributed in our population. Multivariable linear regression analysis was used to examine associations between demographic and socio-economic indicators and nutrients, food groups and the PDQS. Nutrient and food models were adjusted for sex, age and energy intake. We evaluated homogeneity of intakes across subgroups using a trend test across categories of SES, household spending, age category, education and BMI, and a Wald test (with Tukey correction for multiple comparisons by marital status category) for sex, place of residence, 
geographic location and marital status. We did not present data for low-fat dairy and alcoholic beverages due to very low consumption of these food groups in our sample. We detected dietary outliers (none for energy intake and up to twelve extreme values for MUFA, linoleic acid, and vitamins $\mathrm{A}, \mathrm{B}_{12}$ and $\mathrm{C}$ ) using the \%esd macro for performing generalized extreme Studentized deviate many-outlier detection $^{(27)}$. We conducted sensitivity analyses by excluding the extreme values for each log-transformed nutrient and compared the results with those from the full sample. Results were similar with and without inclusion of these outliers and so all observations were included. Due to suspected problems with the validity of the data collected by one of five interviewers, we excluded data from nineteen participants (2\%) whose energy and nutrient intakes were systematically and significantly higher compared with those of the other four interviewers. In a sensitivity analysis, we also adjusted the models for the season effects and compared the results with our initial findings. Sampling weights were included in the models to account for the sampling and participation effects and so that results would be representative of the general adult population in the FB\&H. All the analyses were performed in the statistical software package SAS version 9.4. All $P$ values were two-tailed ( $\alpha=0.05)$.

\section{Results}

\section{Energy and nutrients}

Reported mean energy and macronutrient intakes were similar across all three socio-economic indicators (Table 2 and online supplementary material, Supplemental Table S2). Participants in the high socio-economic group consumed less fibre, $\alpha$-linolenic acid (both $P<0.001$ ), K, Fe, and vitamins $\mathrm{C}$ and $\mathrm{E}$ (all $P=0.01$ ), in models adjusted for sex, age and energy, compared with those in the low and medium socio-economic groups. Participants residing in urban areas had lower fibre $(P=0 \cdot 01)$, all PUFA, linoleic acid $(P=0.01)$, Se, $\mathrm{K}$ and vitamin $\mathrm{E}$ intakes (all $P=0.02$ ) compared with those residing in non-urban areas, while participants from the southern parts of the country reported lower average intakes of fibre, all PUFA, $\alpha$-linolenic acid, $\mathrm{K}$ (all $P<0.001)$, total sugars $(P=0.01)$, linoleic acid $(P=0.03)$, niacin $(P=0.05)$, and vitamins $C$ and $D$ $(P=0.01)$ compared with participants from northern and southern regions.

\section{Foods and food groups}

Women reported higher mean intakes of fruits $(P=0.04)$, and sugar and sweets, and lower intakes of refined grains, red meat (all $P<0.001$ ), potatoes, poultry (both $P=0.05$ ) and processed meats $(P=0.03)$ than men in age- and energy-adjusted models (Table 3 ). Older participants consumed significantly more vegetables $(P<0 \cdot 001)$, vegetable products and whole grains (both $P=0 \cdot 01$ ), fish and seafood $(P<0.001)$, legumes and pulses $(P=0.01)$, and liquid fats and oils $(P=0.01)$, and less sugar and sweets, sugarsweetened beverages (SSB), processed meats (all $P<0.001)$ and solid fats $(P=0.03)$ compared with the youngest age group. Participants from the high-SES group consumed significantly higher mean amounts of whole grains, processed meat (both $P=0.01$ ) and SSB $(P<0.001)$, and significantly lower amounts of vegetables, liquid oils, eggs, fish and seafood (all $P=0 \cdot 01$; Table 4). Participants in urban areas consumed lower quantities of vegetables, legumes and fruit products $(P=0 \cdot 01)$, potatoes $(P=0.02)$ and liquid oils $(P<0.001)$, and more nuts and seeds $(P<0.001)$ compared with those in non-urban areas, while participants from the southern parts of the country consumed less fruits, vegetables, poultry, nuts and seeds (all $P=0.01)$, fruit products $(P=0.04)$, potatoes $(P=0.05)$, high-fat dairy $(P=0.03)$, liquid oils and eggs (both $P<0.001)$, and more processed meats, solid fats, SSB (all $P=0.01)$ and whole grains $(P<0.001)$, compared to people residing in northern and central parts.

\section{Prime Diet Quality Score}

The mean PDQS was 15.8 (Table 5), with a range of 7-28 (out of possible 42 points). There were no differences in diet quality by sex or place of residence. In energy-adjusted models, SES $(P=0.04)$ and tertile of household spending $(P=0.002)$ were inversely associated with the PDQS. These differences did not persist in the multivariable-adjusted models. Diet quality varied strongly by age group, with persons in the highest age group having, on average, a $2 \cdot 5$-point higher PDQS compared with the youngest category $(P<0 \cdot 0001)$. Geographic location was also significantly associated with the PDQS, with participants from the north having a mean PDQS that on average was 1.4 points higher compared with those from the south $(P<0 \cdot 0001)$. Married/cohabitating individuals had a 1.4-point higher PDQS than single participants $(P=0.02)$. Participants in higher BMI categories reported having higher diet quality compared with those with lower BMI $(P=0.04)$. Finally, CVD (in energy-adjusted models, $P=0.009)$ and type 2 diabetes (in multivariable-adjusted models, $P=0.03$ ) were directly associated with diet quality. Evaluation of the contribution of individual fruit and vegetable PDQS components revealed low intakes of dark orange fruits and vegetables (consumers: $n 60$ or $7 \%$ ), dark green leafy vegetables ( $n 138$ or $16 \%$ ) and citrus fruits ( $n 174$ or $20 \%$ ). Overall, our results did not change considerably after adjusting for the season effects (data not shown).

\section{Discussion}

In the present cross-sectional study of diet quality among adults in $\mathrm{B} \& \mathrm{H}$ we found generally poor diet quality as 
Table 2 Socio-economic and geographic variations in nutrient intakes among adults in the Bosnia and Herzegovina dietary survey (2017)

\begin{tabular}{|c|c|c|c|c|c|c|c|c|c|c|c|c|c|c|c|c|c|}
\hline & \multicolumn{7}{|c|}{ Socio-economic status (SES) ${ }^{\star}$} & \multicolumn{5}{|c|}{ Place of residence $†$} & \multicolumn{5}{|c|}{ Geographic location $\ddagger$} \\
\hline & \multicolumn{2}{|c|}{$\begin{array}{l}\text { Low SES } \\
(n 293)\end{array}$} & \multicolumn{2}{|c|}{$\begin{array}{l}\text { Medium SES } \\
(n 498)\end{array}$} & \multicolumn{2}{|c|}{$\begin{array}{l}\text { High SES } \\
(n 62)\end{array}$} & \multirow[b]{2}{*}{$P$ value§ } & \multicolumn{2}{|c|}{$\begin{array}{l}\text { Urban } \\
(n 373)\end{array}$} & \multicolumn{2}{|c|}{$\begin{array}{l}\text { Non-urban } \\
(n 480)\end{array}$} & \multirow[b]{2}{*}{$P$ value§ } & \multicolumn{2}{|c|}{$\begin{array}{l}\text { North } \\
(n 587)\end{array}$} & \multicolumn{2}{|c|}{$\begin{array}{l}\text { South } \\
\text { (n266) }\end{array}$} & \multirow[b]{2}{*}{$P$ value§ } \\
\hline & Mean & SE & Mean & SE & Mean & SE & & Mean & SE & Mean & SE & & Mean & SE & Mean & SE & \\
\hline Energy (kJ) & $8975 \|$ & $281 \cdot 2 \|$ & 9079 & $243 \cdot 1$ & 8113 & 531 & 0.30 & 8820 & $277 \cdot 4$ & 9088 & 223.4 & 0.14 & 8929 & $213 \cdot 4$ & 9054 & $308 \cdot 8$ & 0.84 \\
\hline Energy (kcal) & 2145 & 67.2 & 2170 & 58.1 & 1939 & 127 & 0.30 & 2108 & $66 \cdot 3$ & 2172 & 53.4 & 0.14 & 2134 & 51.0 & 2164 & $73 \cdot 8$ & 0.84 \\
\hline \multicolumn{18}{|l|}{ Macronutrients } \\
\hline Carbohydrate (g) & 267 & 4.0 & 268 & 3.7 & 239 & $10 \cdot 0$ & 0.20 & 267 & 3.2 & 264 & 4.0 & 0.47 & 268 & 2.9 & 260 & $5 \cdot 5$ & 0.27 \\
\hline Protein $(\mathrm{g})$ & 79.1 & 1.4 & $78 \cdot 3$ & 1.0 & $80 \cdot 8$ & 3.6 & 0.77 & 78.8 & $1 \cdot 1$ & 78.8 & 1.2 & 0.90 & 78.9 & 1.0 & 78.7 & 1.5 & 0.69 \\
\hline Total fat $(\mathrm{g})$ & $89 \cdot 6$ & 1.7 & $87 \cdot 1$ & 1.3 & 99.8 & 4.0 & 0.53 & 89.4 & 1.5 & 88.5 & 1.5 & 0.71 & $88 \cdot 6$ & 1.2 & 89.5 & 1.9 & 0.70 \\
\hline Fibre $(\mathrm{g})$ & $25 \cdot 9$ & 0.6 & $22 \cdot 7$ & 0.5 & $18 \cdot 0$ & 1.4 & $<0.001$ & $22 \cdot .1$ & 0.5 & 24.7 & 0.6 & 0.01 & $25 \cdot 2$ & $0 . \overline{5}$ & 20.5 & 0.6 & $<0.001$ \\
\hline Total sugars $(\mathrm{g})$ & $72 \cdot 3$ & 2.9 & 71.4 & 2.5 & $66 \cdot 1$ & $7 \cdot 1$ & 0.85 & $72 \cdot 2$ & $2 \cdot 8$ & $70 \cdot 8$ & 2.5 & 0.63 & $76 \cdot 1$ & $2 \cdot 1$ & 63.1 & 3.2 & 0.01 \\
\hline MUFA (g) & $26 \cdot 6$ & 0.7 & $26 \cdot 4$ & 0.5 & 31.6 & 1.8 & 0.17 & $27 \cdot 2$ & 0.5 & $26 \cdot 5$ & 0.6 & 0.39 & $26 \cdot 7$ & 0.4 & $27 \cdot 0$ & 0.8 & 0.91 \\
\hline SFA $(\mathrm{g})$ & 28.5 & 1.0 & $28 \cdot 6$ & 0.6 & $32 \cdot 2$ & $2 \cdot 1$ & 0.24 & 29.7 & 0.8 & 28.0 & 0.7 & 0.10 & 28.5 & 0.7 & $29 \cdot 2$ & 0.8 & 0.43 \\
\hline PUFA (total) (g) & 23.0 & 0.8 & $19 \cdot 8$ & 0.6 & 20.9 & $2 \cdot 3$ & 0.01 & $20 \cdot 0$ & 0.7 & 22.0 & 0.7 & 0.03 & 22.4 & 0.5 & 18.9 & 0.9 & $<0.001$ \\
\hline PUFA $(n-3)(g)$ & 0.79 & 0.1 & 0.66 & 0.1 & 0.7 & $0 \cdot 1$ & 0.06 & 0.70 & 0.4 & 0.72 & $0 \cdot 1$ & 0.72 & 0.81 & 0.1 & 0.56 & 0.1 & $<0.001$ \\
\hline $\mathrm{DHA}+\mathrm{EPA}(\mathrm{mg})$ & 44.2 & $5 \cdot 5$ & $35 \cdot 7$ & 3.8 & $50 \cdot 7$ & 18.5 & 0.53 & 40.9 & 4.6 & 38.9 & $4 \cdot 3$ & 0.80 & $46 \cdot 7$ & $4 \cdot 3$ & $29 \cdot 0$ & $4 \cdot 2$ & 0.01 \\
\hline$\alpha$-Linolenic acid (mg) & 146 & 29.5 & 68.5 & $15 \cdot 6$ & $17 \cdot 7$ & 16.4 & $<0.001$ & 67.8 & $17 \cdot 7$ & 97.1 & 19.5 & 0.19 & 167 & 14.0 & $22 \cdot 2$ & 9.2 & $<0.001$ \\
\hline Linoleic acid (mg) & $18 \cdot 8$ & 0.7 & 15.4 & 0.6 & $16 \cdot 8$ & $2 \cdot 2$ & 0.01 & $15 \cdot 6$ & 0.6 & $17 \cdot 7$ & 0.7 & 0.02 & 17.5 & 0.5 & 15.4 & 0.9 & 0.03 \\
\hline Cholesterol (mg) & 264 & $10 \cdot 0$ & 255 & $10 \cdot 2$ & 256 & $27 \cdot 0$ & 0.52 & 260 & $11 \cdot 0$ & 257 & $9 \cdot 3$ & 0.79 & 262 & 8.8 & 251 & $12 \cdot 0$ & 0.45 \\
\hline \multicolumn{18}{|l|}{ Minerals } \\
\hline $\mathrm{Ca}(\mathrm{mq})$ & 771 & 24.0 & 766 & $20 \cdot 3$ & 712 & $57 \cdot 0$ & 0.43 & 796 & $19 \cdot 4$ & 740 & 21.5 & 0.05 & 749 & $17 \cdot 7$ & 795 & 28.0 & 0.16 \\
\hline $\mathrm{Mg}(\mathrm{mg})$ & 307 & $6 \cdot 8$ & 291 & $5 \cdot 0$ & 304 & $17 \cdot 3$ & 0.19 & 293 & 4.8 & 303 & $6 \cdot 1$ & 0.20 & 300 & 4.7 & 294 & 8.0 & 0.48 \\
\hline$P(\mathrm{mg})$ & 1147 & $27 \cdot 7$ & 1138 & 21.0 & 1149 & $50 \cdot 0$ & 0.81 & 1157 & 23.4 & 1131 & $22 \cdot 0$ & 0.45 & 1124 & $18 \cdot 3$ & 1177 & 31.0 & 0.17 \\
\hline Se $(\mu \mathrm{g})$ & 72.9 & $2 \cdot 8$ & $71 \cdot 1$ & $2 \cdot 1$ & $60 \cdot 3$ & $5 \cdot 3$ & 0.06 & $67 \cdot 2$ & $2 \cdot 0$ & $74 \cdot 2$ & 2.5 & 0.02 & 73.0 & 1.9 & 67.3 & 3.0 & 0.06 \\
\hline $\mathrm{K}(\mathrm{mg})$ & 3025 & $67 \cdot 0$ & 2809 & $55 \cdot 0$ & 2552 & 170 & 0.01 & 2762 & 63.5 & 2965 & $56 \cdot 1$ & 0.02 & 3007 & $52 \cdot 8$ & 2632 & $66 \cdot 0$ & $<0.001$ \\
\hline $\mathrm{Fe}(\mathrm{mg})$ & 13.4 & 0.3 & $12 \cdot 7$ & 0.3 & 10.7 & 0.7 & 0.01 & 12.5 & 0.3 & 13.1 & 0.3 & 0.18 & 13.1 & 0.2 & $12 \cdot 3$ & 0.4 & 0.07 \\
\hline $\mathrm{Zn}(\mathrm{mg})$ & 9.7 & 0.3 & 10.2 & 0.3 & 8.9 & 0.6 & 0.93 & 9.7 & 0.3 & $10 \cdot 1$ & 0.3 & 0.29 & 9.7 & 0.2 & 10.3 & 0.4 & 0.17 \\
\hline $\mathrm{Na}(\mathrm{mg})$ & 2917 & $65 \cdot 2$ & 2944 & $55 \cdot 1$ & 2940 & 176 & 0.91 & 2908 & $54 \cdot 0$ & 2953 & 59.5 & 0.49 & 2900 & $45 \cdot 9$ & 2999 & $90 \cdot 1$ & 0.37 \\
\hline \multicolumn{18}{|l|}{ Vitamins } \\
\hline$\beta$-Carotene $(\mu \mathrm{g})$ & 1114 & $82 \cdot 6$ & 1018 & 73.9 & 944 & 168 & 0.20 & 982 & $72 \cdot 0$ & 1105 & $76 \cdot 0$ & 0.27 & 1098 & $64 \cdot 7$ & 962 & $90 \cdot 1$ & 0.21 \\
\hline Vitamin A ( $\mu \mathrm{g}$ RE) & 485 & $18 \cdot 6$ & 475 & 23.5 & 413 & 28.7 & 0.17 & 471 & $17 \cdot 0$ & 477 & 23.0 & 0.88 & 494 & $18 \cdot 4$ & 439 & 24.4 & 0.06 \\
\hline Thiamin (mg) & 1.2 & 0.1 & 1.3 & 0.1 & 1.1 & 0.1 & 0.97 & 1.3 & 0.1 & 1.3 & 0.1 & 0.99 & 1.2 & 0.1 & 1.3 & 0.1 & 0.55 \\
\hline Riboflavin (mq) & 1.4 & 0.1 & 1.4 & 0.1 & 1.4 & 0.1 & 0.85 & 1.4 & 0.1 & 1.4 & 0.1 & 0.28 & 1.4 & 0.1 & 1.3 & 0.1 & 0.10 \\
\hline Niacin $(\mathrm{mg})$ & $19 \cdot 8$ & 0.7 & $20 \cdot 6$ & 0.5 & $22 \cdot 8$ & $2 \cdot 6$ & 0.21 & 21.0 & 0.5 & $20 \cdot 0$ & 0.6 & 0.23 & 21.0 & 0.5 & $19 \cdot 3$ & 0.7 & 0.05 \\
\hline Vitamin $B_{6}(\mathrm{mg})$ & 1.3 & 0.1 & 1.4 & 0.1 & 1.4 & 0.1 & 0.43 & 1.3 & 0.1 & 1.3 & 0.1 & 0.63 & 1.4 & 0.1 & 1.3 & 0.1 & 0.38 \\
\hline Vitamin $B_{12}(\mu \mathrm{g})$ & 3.8 & 0.2 & 3.9 & 0.2 & 3.6 & 0.4 & 0.80 & 3.8 & 0.2 & 3.9 & 0.2 & 0.52 & 3.9 & 0.2 & 3.8 & 0.3 & 0.73 \\
\hline Folate $(\mu \mathrm{g})$ & 291 & $12 \cdot \overline{8}$ & 287 & $8 \cdot \overline{6}$ & 283 & 41.3 & 0.73 & 285 & $10 \cdot \overline{3}$ & 291 & 10.0 & 0.67 & 296 & $9 \cdot 1$ & 274 & 13.0 & 0.15 \\
\hline Vitamin C (mg) & 92.5 & $6 \cdot 1$ & 73.2 & 4.2 & 67.2 & $10 \cdot 2$ & 0.01 & 76.0 & 3.9 & 83.3 & $5 \cdot 3$ & 0.28 & 88.8 & 4.3 & 64.9 & $5 \cdot 1$ & 0.01 \\
\hline Vitamin $D(\mu \mathrm{q})$ & 4.1 & 0.3 & 3.3 & 0.2 & 3.6 & 0.5 & 0.03 & 3.8 & 0.2 & 3.5 & 0.2 & 0.35 & 4.0 & 0.2 & 3.1 & 0.2 & 0.01 \\
\hline Vitamin E (mg) & $20 \cdot 3$ & 0.9 & 17.5 & $0 . \overline{6}$ & $16 \cdot 6$ & 1.2 & 0.01 & 17.4 & $0 . \overline{5}$ & 19.4 & $0 . \overline{7}$ & 0.02 & $18 \cdot 7$ & 0.4 & $18 \cdot 2$ & $1 \cdot 1$ & 0.61 \\
\hline
\end{tabular}

'SES is a composite measure composed of the total household consumption category standardized using the Organisation for Economic Co-operation and Development-modified equivalence scale and the highest educational attainment of the head of household.

†'Urban' includes settlements in Bosnia and Herzegovina that have been awarded a 'city' status. 'Non-urban' includes all other settlements, including semi-urban areas.

F North' refers to central and northern parts of the country, mainly Bosnian municipalities; 'south' to Herzegovinian municipalities.

$\S P$ values for homogeneity of means estimated by the linear trend test (SES) and the Wald $F$ test (place of residence, geographic location).

\|Data are presented as geometric means and SE (adjusted for sex, age and energy intake). 
Table 3 Demographic variations in intakes of selected foods among adults in the Bosnia and Herzegovina dietary survey (2017)

\begin{tabular}{|c|c|c|c|c|c|c|c|c|c|c|c|c|}
\hline \multirow[b]{3}{*}{ Food/food group } & \multicolumn{5}{|c|}{ Sex ${ }^{*}$} & \multicolumn{7}{|c|}{ Age group $\dagger$} \\
\hline & \multicolumn{2}{|c|}{$\begin{array}{l}\text { Male } \\
(n 371)\end{array}$} & \multicolumn{2}{|c|}{$\begin{array}{l}\text { Female } \\
(n 482)\end{array}$} & \multirow[b]{2}{*}{$P$ value $\neq$} & \multicolumn{2}{|c|}{$\begin{array}{c}\text { 18-40 years } \\
(n 241)\end{array}$} & \multicolumn{2}{|c|}{$\begin{array}{c}41-60 \text { years } \\
(n 384)\end{array}$} & \multicolumn{2}{|c|}{$\begin{array}{c}\geq 61 \text { years } \\
(n 228)\end{array}$} & \multirow[b]{2}{*}{$P$ value $\neq$} \\
\hline & Mean & SE & Mean & SE & & Mean & SE & Mean & SE & Mean & SE & \\
\hline Fruits $(g / d)$ & 327 & $27 \cdot 8$ & 400 & $22 \cdot 5$ & 0.04 & 324 & $28 \cdot 8$ & 357 & $29 \cdot 7$ & 410 & 31.4 & 0.06 \\
\hline Fruit products $(\mathrm{g} / \mathrm{d})$ & $24 \cdot 7$ & $8 \cdot 3$ & $23 \cdot 0$ & $6 \cdot 0$ & 0.74 & 10.9 & 7.5 & $26 \cdot 3$ & $8 \cdot 1$ & 34.3 & 8.1 & 0.53 \\
\hline Vegetables (g/d) & 271 & $18 \cdot 6$ & 261 & $18 \cdot 8$ & 0.51 & 188 & $16 \cdot 7$ & 266 & $16 \cdot 2$ & 346 & $33 \cdot 8$ & $<0.001$ \\
\hline Vegetable products (g/d) & 54.9 & $5 \cdot 2$ & $55 \cdot 6$ & $5 \cdot 2$ & 0.91 & 47.4 & $7 \cdot 2$ & $48 \cdot 1$ & $8 \cdot 3$ & $71 \cdot 6$ & $12 \cdot 0$ & 0.01 \\
\hline Whole grains $(\mathrm{g} / \mathrm{d})$ & $68 \cdot 3$ & $14 \cdot 1$ & $86 \cdot 7$ & $18 \cdot 5$ & $0 \cdot 10$ & $65 \cdot 2$ & $16 \cdot 2$ & 57.4 & $10 \cdot 0$ & 110 & 18.4 & 0.01 \\
\hline Refined grains (g/d) & 496 & $19 \cdot 2$ & 431 & $21 \cdot 0$ & $<0.001$ & 466 & 23.2 & 477 & $17 \cdot 4$ & 446 & $25 \cdot 9$ & 0.33 \\
\hline Potatoes (g/d) & 141 & $13 \cdot 0$ & 128 & $12 \cdot 0$ & 0.05 & 138 & 13.9 & 116 & $11 \cdot 8$ & 130 & $19 \cdot 8$ & 0.56 \\
\hline Red meat $(\mathrm{g} / \mathrm{d})$ & 153 & $14 \cdot 1$ & 104 & 8.9 & $<0.001$ & 118 & $12 \cdot 6$ & 133 & 11.9 & 134 & $17 \cdot 3$ & 0.41 \\
\hline Processed meat $(\mathrm{g} / \mathrm{d})$ & $65 \cdot 8$ & 6.5 & 53.7 & $6 \cdot 7$ & 0.03 & $78 \cdot 1$ & $8 \cdot 7$ & 59.8 & $7 \cdot 4$ & $41 \cdot 3$ & $6 \cdot 1$ & $<0.001$ \\
\hline Poultry & 102 & $10 \cdot 0$ & $80 \cdot 8$ & $9 \cdot 2$ & 0.05 & 99.0 & 11.3 & 93.4 & $10 \cdot 7$ & 81.3 & $12 \cdot 9$ & 0.23 \\
\hline Fish and seafood (g/d) & $23 \cdot 4$ & $5 \cdot 8$ & $19 \cdot 4$ & $5 \cdot 8$ & $0 \cdot 15$ & $18 \cdot 4$ & $5 \cdot 6$ & $9 \cdot 8$ & 2.5 & $36 \cdot 0$ & $12 \cdot 8$ & $<0.001$ \\
\hline High-fat dairy $(\mathrm{ml} / \mathrm{d}) \S$ & 232 & $19 \cdot 0$ & 247 & $20 \cdot 8$ & 0.59 & 218 & $20 \cdot 8$ & 235 & $22 \cdot 9$ & 266 & $28 \cdot 0$ & $0 \cdot 13$ \\
\hline High-fat dairy $(g / d) \|$ & 103 & 7.6 & 103 & 7.4 & 0.83 & 94.3 & $7 \cdot 7$ & 112 & $8 \cdot 6$ & 102 & $9 \cdot 6$ & 0.45 \\
\hline Low-fat dairy $(\mathrm{ml} / \mathrm{d}) \S$ & $-\eta$ & - & - & - & & - & & - & & - & - & \\
\hline Low-fat dairy $(\mathrm{g} / \mathrm{d}) \|$ & - & - & - & - & & - & & - & & - & - & \\
\hline Nuts and seeds $(\mathrm{g} / \mathrm{d})$ & 7.9 & $2 \cdot 0$ & 8.5 & $1 \cdot 3$ & 0.95 & $9 \cdot 7$ & $1 \cdot 8$ & $5 \cdot 8$ & $1 \cdot 3$ & $9 \cdot 1$ & $2 \cdot 7$ & 0.25 \\
\hline Legumes and pulses (g/d) & 33.4 & $6 \cdot 8$ & $31 \cdot 1$ & $5 \cdot 2$ & 0.52 & 24.9 & $6 \cdot 0$ & $26 \cdot 3$ & $4 \cdot 1$ & 45.5 & 8.9 & 0.01 \\
\hline Eggs (g/d) & $67 \cdot 0$ & 6.9 & 64.4 & $5 \cdot 2$ & 0.43 & $61 \cdot 8$ & $8 \cdot 3$ & $67 \cdot 4$ & 5.9 & $67 \cdot 8$ & 6.5 & 0.50 \\
\hline Sugar and sweets $(\mathrm{g} / \mathrm{d})$ & $81 \cdot 2$ & 6.5 & $107 \cdot 3$ & 6.5 & $<0.001$ & 131 & $9 \cdot 7$ & $76 \cdot 6$ & $5 \cdot 7$ & $75 \cdot 6$ & $7 \cdot 3$ & $<0.001$ \\
\hline Solid fats $(g / d)$ & $13 \cdot 1$ & 1.8 & $13 \cdot 0$ & $1 \cdot 7$ & 0.85 & $15 \cdot 3$ & $2 \cdot 3$ & 11.5 & 1.8 & $12 \cdot 2$ & $2 \cdot 1$ & 0.03 \\
\hline Liquid fats and oils (ml) & $42 \cdot 1$ & 1.9 & $44 \cdot 1$ & $2 \cdot 0$ & 0.08 & $39 \cdot 7$ & $2 \cdot 3$ & $43 \cdot 8$ & $2 \cdot 1$ & 45.9 & $2 \cdot 6$ & 0.01 \\
\hline Sugar-sweetened beverages (ml) & 167 & $26 \cdot 0$ & 169 & $22 \cdot 8$ & 0.99 & 280 & 33.8 & 113 & $18 \cdot 4$ & 108 & $27 \cdot 1$ & $<0.001$ \\
\hline
\end{tabular}

${ }^{*}$ Age- and energy-adjusted mean and SE.

†Sex- and energy-adjusted mean and SE.

$\ddagger P$ values for homogeneity estimated by the Wald $\chi^{2}$ and a trend test for subgroup effect with a Gamma distribution

$\S$ Milk, yoghurt and other dairy drinks.

\|Solid dairy products (except butter and ghee)

qFoods consumed by very few participants.

measured by the PDQS across all subgroups of our sample, and this was largely characterized by low intakes of nutrient-rich fruits and vegetables, low-fat dairy, fish, and nuts and seeds, and high intakes of processed meats and refined grains. We also observed that the diet quality of Bosnian adults varied significantly by age, geographic location, BMI, type 2 diabetes status and marital status, but contrary to our initial hypothesis, did not vary by markers of SES.

Our results regarding all three levels of analysis, including nutrients, food groups and dietary pattern, suggested that diet quality among young and middle-aged persons is lower compared with those in the older age group. One possible explanation for this finding is that older participants may have changed their diet as a result of disease, not to prevent it, given the high prevalence of chronic disease in this sample. However, our results did not change after adjusting for chronic disease in our models. An alternative explanation could be that older individuals are more immune to the nutrition transition occurring in the country compared with younger people. We also found significant differences in overall diet quality by geographic location, with the participants living in the south having poorer dietary habits than those living in northern and central parts. Low intakes of $n-3$ fatty acids in this group were especially worrisome given their role in prevention of $\mathrm{CVD}^{(28,29)}$. This is surprising, as participants from the south would be expected to have more access to fresh produce and fish and dietary habits that are thought to resemble those of a Mediterranean diet. However, studies ${ }^{(14,30)}$ have found similar changes in dietary habits in other Mediterranean areas, where olive oil and fish were being replaced by solid fats and red meat. Also, the HBS $2007^{(17)}$ found that households that produced fruits and vegetables had significantly lower consumption of fresh produce compared with those that purchased them. Therefore, a possible explanation for our findings could be that participants living in the south tend to sell the fruits and vegetables they produce to purchase less healthy foods, such as SSB and processed meats, for their own consumption.

Diet quality measured by the PDQS was inversely associated with SES in the energy-adjusted models, but these associations were no longer significant after adjusting for covariates. While the PDQS was slightly higher as the level of education of the head of household increased, diet quality score was lower with higher household spending. Our SES findings can, to some extent, be explained by the socioeconomic history of the country. First, correlations between education level and wealth have traditionally not been as 
Table 4 Socio-economic variations in intakes of selected foods among adults in the Bosnia and Herzegovina dietary survey (2017)

\begin{tabular}{|c|c|c|c|c|c|c|c|c|c|c|c|c|c|c|c|c|c|}
\hline \multirow[b]{3}{*}{ Food/food group } & \multicolumn{7}{|c|}{ Socio-economic status (SES) ${ }^{\star}$} & \multicolumn{5}{|c|}{ Place of residence ${ }^{*}$} & \multicolumn{5}{|c|}{ Geographic location* } \\
\hline & \multicolumn{2}{|c|}{$\begin{array}{c}\text { Low SES } \\
(n 293)\end{array}$} & \multicolumn{2}{|c|}{$\begin{array}{l}\text { Medium SES } \\
(n 498)\end{array}$} & \multicolumn{2}{|c|}{$\begin{array}{l}\text { High SES } \\
\quad(n 62)\end{array}$} & \multirow[b]{2}{*}{$P$ value $†$} & \multicolumn{2}{|c|}{$\begin{array}{l}\text { Urban } \\
(n 373)\end{array}$} & \multicolumn{2}{|c|}{$\begin{array}{l}\text { Other } \\
(n 480)\end{array}$} & \multirow[b]{2}{*}{$P$ value† } & \multicolumn{2}{|c|}{$\begin{array}{l}\text { North } \\
(n 587)\end{array}$} & \multicolumn{2}{|c|}{$\begin{array}{l}\text { South } \\
(n 266)\end{array}$} & \multirow[b]{2}{*}{$P$ value† } \\
\hline & Mean & SE & Mean & SE & Mean & SE & & Mean & SE & Mean & SE & & Mean & SE & Mean & SE & \\
\hline Fruits $(g / d)$ & 382 & $30 \cdot 2$ & 357 & $22 \cdot 2$ & 292 & $55 \cdot 2$ & 0.20 & 388 & $25 \cdot 1$ & 342 & 23.8 & 0.15 & 406 & $22 \cdot 0$ & 280 & $25 \cdot 0$ & 0.01 \\
\hline Fruit products $(\mathrm{g} / \mathrm{d})$ & 33.0 & $10 \cdot 2$ & $19 \cdot 4$ & $5 . \overline{9}$ & $12 \cdot 6$ & 5.4 & 0.66 & $12 \cdot 9$ & 3.5 & $33 \cdot 1$ & 8.7 & 0.01 & 29.5 & 6.9 & 13.9 & $6 \cdot 8$ & 0.04 \\
\hline Vegetables (g/d) & 297 & $23 \cdot 7$ & 245 & 14.5 & 241 & $20 \cdot 6$ & 0.01 & 238 & $15 \cdot 6$ & 285 & $18 \cdot 1$ & 0.01 & 279 & $17 \cdot 4$ & 237 & $14 \cdot 6$ & 0.01 \\
\hline Vegetable products $(\mathrm{g} / \mathrm{d})$ & 53.8 & $8 \cdot 0$ & 54.0 & $7 \cdot 1$ & $60 \cdot 0$ & 11.7 & 0.76 & 53.0 & $6 \cdot 4$ & $55 \cdot 1$ & 7.4 & 0.65 & $56 \cdot 0$ & $6 \cdot 2$ & $50 \cdot 8$ & $9 \cdot 3$ & 0.53 \\
\hline Whole grains $(\mathrm{g} / \mathrm{d})$ & $71 \cdot 0$ & $15 \cdot 4$ & $73 \cdot 1$ & 10.9 & 110 & $22 \cdot 0$ & 0.01 & $71 \cdot 6$ & 7.9 & 77.4 & $13 \cdot 8$ & 0.42 & $59 \cdot 1$ & 8.7 & 105 & $21 \cdot 4$ & $<0.001$ \\
\hline Refined grains $(\mathrm{g} / \mathrm{d})$ & 463 & $16 \cdot 7$ & 477 & $17 \cdot 7$ & 385 & $43 \cdot 0$ & 0.58 & 464 & $16 \cdot 0$ & 466 & $17 \cdot 4$ & 0.27 & 458 & $12 \cdot 1$ & 479 & $27 \cdot 8$ & 0.12 \\
\hline Potatoes $(\mathrm{g} / \mathrm{d})$ & 136 & 13.5 & 126 & 11.5 & $72 \cdot 2$ & $16 \cdot 4$ & 0.06 & 109 & $12 \cdot 4$ & 139 & 11.0 & 0.02 & 136 & 11.2 & 107 & 11.1 & 0.05 \\
\hline Red meat $(g / d)$ & 123 & 12.5 & 131 & $10 \cdot 4$ & 150 & $35 \cdot 0$ & 0.19 & 124 & $10 \cdot 6$ & 133 & 11.3 & $0 \cdot 16$ & 124 & 8.9 & 139 & $14 \cdot 9$ & $0 \cdot 10$ \\
\hline Processed meat $(\mathrm{g} / \mathrm{d})$ & $45 \cdot 3$ & $7 \cdot 3$ & $67 \cdot 6$ & $5 \cdot 8$ & $77 \cdot 7$ & $15 \cdot 8$ & 0.01 & $69 \cdot 8$ & $6 \cdot 0$ & $51 \cdot 6$ & $6 \cdot 4$ & 0.19 & 49.5 & $5 \cdot 2$ & $78 \cdot 8$ & $8 \cdot 3$ & 0.01 \\
\hline Poultry & 98 & 11.6 & $84 \cdot 1$ & 8.4 & 104 & $19 \cdot 8$ & 0.51 & $85 \cdot 0$ & $8 \cdot 0$ & $95 \cdot 8$ & $9 \cdot 8$ & 0.22 & 104 & $8 \cdot 2$ & $65 \cdot 6$ & 10.5 & 0.01 \\
\hline Fish and seafood $(\mathrm{g} / \mathrm{d})$ & $25 \cdot 4$ & 8.9 & $15 \cdot 4$ & $3 \cdot 3$ & $19 \cdot 8$ & $11 \cdot 0$ & 0.01 & 18.9 & 4.5 & $20 \cdot 2$ & $6 \cdot 1$ & 0.75 & 21.4 & $5 \cdot 7$ & $16 \cdot 3$ & $4 \cdot 3$ & 0.33 \\
\hline High-fat dairy $(\mathrm{ml} / \mathrm{d}) \ddagger$ & 267 & $23 \cdot 0$ & 228 & $17 \cdot 7$ & 192 & $30 \cdot 8$ & 0.04 & 233 & $17 \cdot 8$ & 247 & 19.5 & 0.20 & 258 & $16 \cdot 6$ & 207 & $22 \cdot 6$ & 0.03 \\
\hline High-fat dairy $(\mathrm{g} / \mathrm{d}) \S$ & 109 & 8.9 & 102 & $6 \cdot 3$ & 88.2 & 14.0 & 0.19 & 110 & $7 \cdot 1$ & 99.0 & 6.9 & 0.49 & 104 & 6.4 & 103 & $8 \cdot 1$ & 0.70 \\
\hline Low-fat dairy $(\mathrm{ml} / \mathrm{d}) \ddagger$ & $-\|$ & & - & & - & & & - & & - & & & - & & - & & \\
\hline Low-fat dairy $(\mathrm{g} / \mathrm{d}) \S$ & - & & - & & - & & & - & & - & & & - & & - & & \\
\hline Nuts and seeds $(\mathrm{g} / \mathrm{d})$ & $8 \cdot 2$ & 1.7 & 6.6 & 1.2 & $16 \cdot 0$ & 8.5 & 0.12 & $10 \cdot 4$ & $2 \cdot 0$ & $6 \cdot 0$ & 1.2 & $<0.001$ & 9.2 & 1.3 & 5.4 & $2 \cdot 1$ & 0.01 \\
\hline Legumes and pulses $(\mathrm{g} / \mathrm{d})$ & 33.5 & $5 \cdot 6$ & $30 \cdot 3$ & $5 \cdot 3$ & $29 \cdot 0$ & $13 \cdot 1$ & 0.44 & 24.4 & 4.0 & $37 \cdot 0$ & $5 \cdot 8$ & 0.01 & $30 \cdot 5$ & $4 \cdot 1$ & 33.4 & 7.4 & 0.28 \\
\hline Eggs $(g / d)$ & $73 \cdot 8$ & $7 \cdot 3$ & $62 \cdot 6$ & $5 \cdot 3$ & $45 \cdot 9$ & 8.4 & 0.01 & $65 \cdot 2$ & $5 \cdot 6$ & $66 \cdot 3$ & $5 \cdot 8$ & 0.63 & 74.0 & 5.4 & $50 \cdot 0$ & 5.9 & $<0.001$ \\
\hline Sugar and sweets $(g / d)$ & $88 \cdot 8$ & $6 \cdot 8$ & 94.2 & $6 \cdot 3$ & 107 & $17 \cdot 4$ & 0.06 & 103 & $8 \cdot 0$ & 85.4 & 4.8 & 0.15 & $98 \cdot 1$ & $5 \cdot 6$ & $82 \cdot 7$ & 7.5 & 0.85 \\
\hline Solid fats $(g / d)$ & $12 \cdot 6$ & 1.6 & $12 \cdot 3$ & 0.9 & $21 \cdot 0$ & $5 \cdot 0$ & 0.05 & $14 \cdot 2$ & $2 \cdot 0$ & 11.9 & 1.6 & 0.09 & $11 \cdot 2$ & $1 \cdot 3$ & $16 \cdot 0$ & $2 \cdot 4$ & 0.01 \\
\hline Liquid fats and oils (ml) & $51 \cdot 0$ & $2 \cdot 3$ & $38 \cdot 3$ & 1.4 & $37 \cdot 7$ & 3.9 & $<0.001$ & $38 \cdot 1$ & 1.4 & $47 \cdot 2$ & 1.9 & $<0.001$ & $47 \cdot 0$ & $1 \cdot 6$ & 35.9 & 1.9 & $<0.001$ \\
\hline Sugar-sweetened beverages (ml) & 132 & $23 \cdot 1$ & 179 & $23 \cdot 6$ & $195 \cdot 8$ & $40 \cdot 7$ & 0.01 & 172 & $18 \cdot 3$ & 155 & 14.5 & 0.94 & 149 & 20.5 & 189 & $27 \cdot 7$ & 0.01 \\
\hline
\end{tabular}

*Age-, energy- and sex-adjusted mean and SE.

t $P$ values for homogeneity of means estimated by the Wald $\chi^{2}$ test and a trend test for subgroup effect with a Gamma distribution $\ddagger$ Milk, yoghurt and other dairy drinks.

§Solid dairy products (except butter and ghee).

$\|$ Foods consumed by very few participants. 
Table 5 Predictors of diet quality measured by the Prime Diet Quality Score (PDQS) among adults in Bosnia and Herzegovina (2017)

\begin{tabular}{|c|c|c|c|c|}
\hline \multirow[b]{2}{*}{ Characteristic } & \multicolumn{2}{|c|}{$\begin{array}{l}\text { PDQS, energy-adjusted } \\
(n 836)\end{array}$} & \multicolumn{2}{|c|}{$\begin{array}{l}\text { PDQS, covariate-adjusted } \\
(n 836)^{\star}\end{array}$} \\
\hline & Mean & SE & Mean & SE \\
\hline \multicolumn{5}{|l|}{ Sex } \\
\hline Male & $15 \cdot 8$ & 0.2 & $15 \cdot 7$ & 0.3 \\
\hline Female & $15 \cdot 8$ & 0.2 & $15 \cdot 9$ & 0.3 \\
\hline$P$ value for sex effect $\dagger$ & 0.9 & & 0.52 & \\
\hline \multicolumn{5}{|l|}{ Age group } \\
\hline $18-40$ years & $14 \cdot 7$ & 0.3 & 14.5 & 0.3 \\
\hline $41-60$ years & $16 \cdot 0$ & 0.2 & $16 \cdot 0$ & 0.3 \\
\hline$\geq 61$ years & $17 \cdot 2$ & 0.3 & $17 \cdot 0$ & 0.4 \\
\hline $\bar{P}$ value for age effect $\dagger$ & $<0.0001^{*}$ & & $<0.0001^{\star}$ & \\
\hline \multicolumn{5}{|l|}{ Socio-economic status (SES) } \\
\hline Low SES & $16 \cdot 2$ & 0.2 & $15 \cdot 9$ & 0.3 \\
\hline Medium SES & $15 \cdot \overline{5}$ & 0.2 & $15 \cdot 5$ & 0.2 \\
\hline High SES & $15 \cdot 4$ & 0.7 & $16 \cdot 1$ & 0.7 \\
\hline$P$ value for SES effect $†$ & $0.04^{*}$ & & 0.57 & \\
\hline \multicolumn{5}{|l|}{ Household spending } \\
\hline Tertile 1 (lowest) & $16 \cdot 2$ & 0.2 & $16 \cdot 2$ & 0.3 \\
\hline Tertile 2 & $16 \cdot 0$ & 0.2 & $16 \cdot 0$ & 0.3 \\
\hline Tertile 3 & $15 \cdot 1$ & 0.2 & $15 \cdot 5$ & 0.3 \\
\hline$P$ value for household spending effect $\dagger$ & $0.002^{*}$ & & 0.09 & \\
\hline \multicolumn{5}{|l|}{ Educational attainment of the household head } \\
\hline$<12$ years & $16 \cdot 0$ & 0.3 & $15 \cdot 5$ & 0.3 \\
\hline 12 years & $15 \cdot 7$ & 0.2 & $15 \cdot 6$ & 0.2 \\
\hline$>12$ years & $16 \cdot 0$ & 0.6 & $16 \cdot 6$ & 0.5 \\
\hline$P$ value for education effect $\dagger$ & 0.49 & & 0.86 & \\
\hline \multicolumn{5}{|l|}{ Place of residence } \\
\hline Urban & $15 \cdot 7$ & 0.2 & $15 \cdot 8$ & 0.3 \\
\hline Non-urban & $15 \cdot 9$ & 0.2 & $15 \cdot 8$ & 0.3 \\
\hline$P$ value for education effect $\dagger$ & 0.55 & & 0.90 & \\
\hline \multicolumn{5}{|l|}{ Geographic location } \\
\hline North (Bosnia) & $16 \cdot 3$ & 0.2 & $16 \cdot 5$ & 0.3 \\
\hline South (Herzegovina) & $14 \cdot 8$ & 0.3 & $15 \cdot 1$ & 0.3 \\
\hline$P$ value for geographic location effect $\dagger$ & $<0.0001^{\star}$ & & $<0.0001^{\star}$ & \\
\hline \multicolumn{5}{|l|}{$\mathrm{BMl}\left(\mathrm{kg} / \mathrm{m}^{2}\right)$} \\
\hline$<25.0$ & $15 \cdot 2$ & 0.3 & $15 \cdot 5$ & 0.3 \\
\hline $25 \cdot 0-29 \cdot 9$ & $15 \cdot 5$ & 0.2 & $15 \cdot 6$ & 0.3 \\
\hline$\geq 30 \cdot 0$ & $17 \cdot 2$ & 0.3 & $16 \cdot 6$ & 0.4 \\
\hline$P$ value for BMI effect $\dagger$ & $<0.0001^{*}$ & & $0.04^{*}$ & \\
\hline \multicolumn{5}{|l|}{ Marital status } \\
\hline Single & $14 \cdot 0$ & 0.4 & $14 \cdot 8$ & 0.5 \\
\hline Married & $16 \cdot 1$ & 0.2 & $16 \cdot 1$ & 0.3 \\
\hline Divorced/widowed & $16 \cdot 7$ & 0.4 & $15 \cdot 7$ & 0.4 \\
\hline$P$ value for marital status effect $†$ & $<0.0001^{\star}$ & & $0.02^{*}$ & \\
\hline \multicolumn{5}{|l|}{ CVD } \\
\hline Yes & $16 \cdot 6$ & 0.3 & $15 \cdot 7$ & 0.4 \\
\hline No & $15 \cdot 6$ & 0.2 & $15 \cdot 9$ & 0.3 \\
\hline$P$ value for CVD status effect $†$ & $0.009^{*}$ & & 0.71 & \\
\hline \multicolumn{5}{|l|}{ Type 2 diabetes (T2D) } \\
\hline Yes & $17 \cdot 6$ & 0.5 & $16 \cdot 8$ & 0.5 \\
\hline No & $15 \cdot 7$ & 0.2 & $15 \cdot 7$ & 0.3 \\
\hline$P$ value for T2D status effect + & $<0.0001$ & & $0.03^{*}$ & \\
\hline
\end{tabular}

*Adjusted for total energy intake (continuous), sex (male, female), age group (18-40, 41-60, $\geq 61$ years), SES (tertiles; except for education subgroups, which are adjusted for tertile of household spending, and household spending subgroups, which are adjusted for the educational attainment of the head of household ( $<12$ years, 12 years, $>12$ years)), place of residence (urban, non-urban) and geographic location (Bosnia/north or Herzegovina/south).

$\dagger P$ values for homogeneity estimated by the Wald $F$ test (for sex, place of residence, geographic location and marital status, using Tukey correction to adjust for multiple comparisons for marital status) and trend test (for age, SES, household spending, education and BMI) in multivariate linear regression analyses, adjusted for covariates listed above.

strong in socialist countries as in capitalist economies ${ }^{(31,32)}$. Second, an economic transition from a planned to market economy in the 1990s followed by 4 years of armed conflict led to major demographic and economic changes in form of internal and external migrations, loss of wealth and the post-war accumulation of profits among less-educated groups $^{(33)}$. Lower diet quality among persons in the high
SES category is in line with the nutrition transition patterns observed in low- and middle-income countries where highincome individuals are first to be hit by the transition ${ }^{(6)}$. Recent internal migrations could explain why the present study did not find any significant differences in rural $v$. urban overall diet quality. It is plausible that, as people migrated from rural to urban areas leaving lands behind 
and accumulating wealth, the adoption of Western dietary patterns increased ${ }^{(4,34)}$. As the nutrition transition in the country progresses, however, it may become more evident that the urban poor are at highest risk of eating poor diets and having adverse health outcomes, as documented over the past few decades in other countries ${ }^{(35)}$.

Higher diet quality score among married/cohabitating participants reaffirms the importance of marital status in promoting healthy dietary behaviour ${ }^{(36,37)}$. Together with the data on diet quality variation by age group it also underlines the importance of teaching cooking skills to the younger population so that they will be able to prepare their own healthy meals when they leave the family home ${ }^{(38)}$. Obese individuals in our cohort tended to report eating healthier diets compared with those with a normal BMI. One possible explanation for this is that obese individuals made dietary changes in order to lose weight. Since our study was cross-sectional, these findings may be prone to reverse causality ${ }^{(39)}$. Alternatively, it is entirely possible that people with higher BMI tended to have higher PDQS because they tended to eat larger quantities of food from all the food groups. While we attempted to control for total energy intake to account for this bias, there may be residual confounding that is still driving this association. Finally, it does not surprise that persons with diabetes had higher diet quality scores compared with healthy individuals given dietary modifications are a part of routine medical treatment for this condition ${ }^{(40)}$.

While more work is needed before the PDQS is validated for use with $24 \mathrm{~h}$ recall data and as a standalone data collection tool, it is promising to see that it is in concordance with the nutrient-based and food-based findings. For example, participants in the highest age group reported consuming more vegetables, whole grains, fish, legumes and oils, and less processed meat, solid fats, sweets and SSB, and were awarded significantly more PDQS points compared with younger participants. They also reported lower intakes of sugar, $\mathrm{K}, \mathrm{Na}$ and $\mathrm{Ca}$ and higher intakes of $\beta$-carotene compared with younger groups ${ }^{(25)}$. Similarly, participants living in the south reported consuming less fibre, $n-3$ and $n$-6 fatty acids, $\mathrm{K}$, thiamin, niacin, and vitamins $\mathrm{C}$ and $\mathrm{D}$, fruit and vegetables, poultry, nuts and oils, and more processed meats, solid fats and SSB than those living in the northern and central parts of the country, and the PDQS was able to distinguish between these two groups well.

Food-based analysis indicated very high intakes of refined grains (white bread, pastries and rice), high-fat dairy, processed meats and low intakes of whole grains, nuts and fish across all groups. Mean intakes of fruits and vegetables were above the WHO recommendation ${ }^{(41)}$ and liquid oils were used more than solid fats. This, however, does not necessarily imply adequate intakes among all participants. The analysis of the individual PDQS fruit and vegetable components indicated the need for consuming a higher variety of fruits and vegetables by including more citrus fruits, dark green leafy vegetables, and dark orange fruits and vegetables. Nutrient-based analysis suggested that mean intakes of some of the key nutrients commonly found in fruits, vegetables and seafood (e.g. fibre among high SES, $\beta$-carotene, vitamin $\mathrm{C}$ and all PUFA) ${ }^{(42,43)}$ were below the WHO recommendations, while intakes of SFA and Na were beyond recommended intakes ${ }^{(41,44)}$; these data support our findings using the PDQS.

Limitations of our study should be considered. First, we inevitably have some level of measurement error in both dietary and socio-economic variables, as both were based on self-report. This source of error is, however, expected to be largely random, producing valid estimates for the population as a whole just with somewhat higher SE. While we did find a systematic error by one of the interviewers (who collected data from nineteen persons before leaving the study) and excluded these observations from our analysis, our findings, with the exception of mean energy intakes, did not change notably. Second, the education level of the female who is usually in charge of food preparation may be more relevant than that of a male head of household ${ }^{(3)}$. Unfortunately, we were unable to evaluate the impact of this variable on participant diet quality as there were too few female participants in our sample with high levels of education. Among the strengths of the present study is that it links standard socio-economic data with dietary data and allows analysis of diet quality by SES indicators. Further, it included a sample representative of the FB\&H's adult population and a sample similar to the B\&H's general adult population across multiple strata (see online supplementary material, Supplemental Table S3). Thus, the study findings may be generalized to the whole population of adult men and non-pregnant, non-breastfeeding women in FB\&H, and likely to the entire $\mathrm{B} \& \mathrm{H}$. While the cross-sectional nature of the study did not allow for a longitudinal evaluation of these indicators on dietary intakes and diet quality, it will serve as an anchor for future trend analyses once several cycles of dietary intakes are obtained.

Low diet quality across all demographic and socioeconomic indicators was evident in this sample of adults from $\mathrm{B} \& \mathrm{H}$, with even lower quality diet scores recorded among younger participants and those living in the south. Overall diet quality score could be improved by incorporating into meals some locally available, highly nutritious, yet in our sample neglected foods such as carrots, collard greens, trout, sardines, mackerel, walnuts, rye and buckwheat. Future research should address reasons behind dietary choices among populations identified as being at elevated risk of inadequate diets.

\section{Acknowledgements}

Financial support: This work was supported by the Nutrition Department of the Harvard T.H. Chan School of Public Health and the Institute of Statistics of the Federation of Bosnia and Herzegovina, who had no role 
in the design, analysis or writing of this article. Conflict of interest: None. Authorship: S.G.: manuscript writing, data collection, data analysis; A.J.K.: data analysis, manuscript editing; T.T.F.: study design, data analysis, manuscript editing; B.R.: study design, data analysis, manuscript editing; E.K.: funds acquisition, data collection, manuscript editing; E.S.: data analysis; A.K.: data analysis; J.M.: data collection, data analysis, manuscript editing; W.W.: study design and supervision, funds acquisition, final manuscript approval. Ethics of human subject participation: This study was conducted according to the guidelines laid down in the Declaration of Helsinki and all procedures involving research study participants were approved by the Ethics Committee of the Public Health Institute of the Federation of Bosnia and Herzegovina and the Harvard T.H. Chan School of Public Health Office of Human Research Administration. Written informed consent was obtained from all participants.

\section{Supplementary material}

To view supplementary material for this article, please visit https://doi.org/10.1017/S1368980019001988

\section{References}

1. Wang DD, Leung CW, Li Y et al. (2014) Trends in dietary quality among adults in the United States, 1999 through 2010. JAMA Intern Med 174, 1587-1595.

2. Popkin BM, Siega-Riz AM \& Haines PS (1996) A comparison of dietary trends among racial and socioeconomic groups in the United States. N Engl J Med 335, 716-720.

3. Beghin L, Dauchet L, De Vriendt T et al. (2014) Influence of parental socio-economic status on diet quality of European adolescents: results from the HELENA study. $\mathrm{Br} J$ Nutr 111, 1303-1312.

4. Popkin BM (2001) The nutrition transition and obesity in the developing world. J Nutr 131, issue 3, 871S-873S.

5. Popkin BM (2004) The nutrition transition: an overview of world patterns of change. Nutr Rev 62, S140-S143.

6. Popkin BM (2014) Synthesis and implications: China's nutrition transition in the context of changes across other low- and middle-income countries. Obes Rev 15, Suppl. 1, 60-67.

7. Mayen AL, Marques-Vidal P, Paccaud F et al. (2014) Socioeconomic determinants of dietary patterns in low- and middle-income countries: a systematic review. Am J Clin Nutr 100, 1520-1531.

8. World Bank (2004) Bosnia and Herzegovina: Post-Conflict Reconstruction and the Transition to a Market Economy. Washington, DC: World Bank.

9. Stiblar F (2013) Economic Growth and Development in Post Yugoslav Countries. Global Europe Program. Washington, DC: Wilson Center.

10. Food and Agriculture Organization of the United Nations (2015) The Fisheries and Aquaculture Sector in Bosnia and Herzegovina: Preparation of IPARD Forest and Fisheries Sector Reviews in Bosnia and Herzegovina. Budapest: FAO Regional Office for Europe and Central Asia.

11. Farrugio H \& Soldo A (2014) Status and Conservation of Fisheries in the Adriatic Sea. Draft internal report for the purposes of the Mediterranean Regional Workshop to Facilitate the Description of Ecologically or Biologically Significant Marine Areas, Malaga, Spain, 7-11 April 2014. United Nations Environment Programme, Mediterranean Action Plan and Regional Activity Centre for Specially Protected Areas.

12. World Health Organization (2015) Bosnia and Herzegovina: WHO Statistical Profile 2012. Geneva: WHO.

13. Public Health Institute FB\&H (2012) Health Population Survey in the Federation of Bosnia and Herzegovina. Sarajevo: Public Health Institute FB\&H.

14. Buzina R, Suboticanec K \& Saric M (1991) Diet patterns and health problems: diet in southern Europe. Ann Nutr Metab 35, Suppl. 1, 32-40.

15. Buzina R, Ferber E, Keys A et al. (1964) Diets of rural families and heads of families in two regions of Yugoslavia. Voeding 25, 629-639.

16. Jonsson IM, Wallin AM, Hallberg LR et al. (2002) Choice of food and food traditions in pre-war Bosnia-Herzegovina: focus group interviews with immigrant women in Sweden. Ethn Health 7, 149-161.

17. Agency for Statistics of Bosnia and Herzegovina (2015) Bosnia and Herzegovina Household Budget Survey. http:// www.bhas.ba/ankete/TB_HBS\%202015_SR.pdf (accessed July 2019).

18. Merten C, Ferrari P, Banner M et al. (2011) Methodological characteristics of the national dietary surveys carried out in the European Union as included in the European Food Safety Authority (EFSA) Comprehensive European Food Consumption Database. Food Addit Contam Part A Chem Anal Control Expo Risk Assess 28, 975-995.

19. Hagenaars A, de Vos K \& Zaidi MA (1994) Poverty Statistics in the Late 1980s: Research Based on Micro-data. Luxembourg: Office for Official Publications of the European Communities.

20. Gurinovic M, Milesevic J, Kadvan A et al. (2016) Establishment and advances in the online Serbian food and recipe data base harmonized with EuroFIR standards. Food Chem 193, 30-38.

21. Nikolić M, Milešević J, Zeković M et al. (2018) The development and validation of food atlas for portion size estimation in the Balkan region. Front Nutr 5, 78.

22. Gurinović M, Milešević J, Kadvan A et al. (2018) Development, features and application of DIET ASSESS \& PLAN (DAP) software in supporting public health nutrition research in Central Eastern European Countries (CEEC). Food Chem 238, 186-194.

23. Fung TT, Isanaka S, Hu FB et al. (2018) International food group-based diet quality and risk of coronary heart disease in men and women. Am J Clin Nutr 107, 120-129.

24. Gicevic S, Gaskins AJ, Fung TT et al. (2018) Evaluating pre-pregnancy dietary diversity vs. dietary quality scores as predictors of gestational diabetes and hypertensive disorders of pregnancy. PLoS One 13, e0195103.

25. Gicevic S, Gaskins AJ, Fung TT et al. (2019) Fueling an epidemic of non-communicable disease in the Balkans: a nutritional survey of Bosnian adults. Int J Public Health 64, 873-885.

26. Gicevic S, Kremic E, Fung TT et al. (2019) Feasibility and sustainability of dietary surveillance, Bosnia and Herzegovina. Bull World Health Organ 97, 349-357.

27. Rosner B (1983) Percentage points for a generalized ESD many-outlier procedure. Technometrics 25, 165-172.

28. Willett WC (2012) Dietary fats and coronary heart disease. J Intern Med 272, 13-24.

29. Willett WC \& Stampfer MJ (2013) Current evidence on healthy eating. Annu Rev Public Health 34, 77-95.

30. Tur JA, Romaguera D \& Pons A (2004) Food consumption patterns in a Mediterranean region: does the Mediterranean diet still exist? Ann Nutr Metab 48, 193-201. 
31. Carnevale AP, Rose SJ \& Cheah B (2015) The College Payoff: Education, Occupations, and Lifetime Earnings. Washington, DC: Georgetown University Center on Education and the Workforce.

32. Boshara R, Emmons WR \& Noeth B (2015) The demographics of wealth: how age, education and race separate thrivers from strugglers in today's economy. Essay No. 2: The role of education. Demogr Wealth issue 2, 1-28.

33. Sorensen JS (2005) War as social transformation: wealth, class, power and an illiberal economy in Serbia. Civil Wars 6, 55-82.

34. De Haen H, Stamoulis K, Shetty P et al. (2004) The world food economy in the twenty-first century: challenges for international co-operation. Dev Policy Rev 21, 683-696.

35. Monsivais P, McLain J \& Drewnowski A (2010) The rising disparity in the price of healthful foods: 2004-2008. Food Policy 35, 514-520.

36. Lee S, Cho E, Grodstein F et al. (2005) Effects of marital transitions on changes in dietary and other health behaviours in US women. Int J Epidemiol 34, 69-78.

37. Eng PM, Kawachi I, Fitzmaurice G et al. (2005) Effects of marital transitions on changes in dietary and other health behaviours in US male health professionals. $J$ Epidemiol Community Health 59, 56-62.
38. da Rocha Leal FM, de Oliveira BMPM \& Pereira SSR (2011) Relationship between cooking habits and skills and Mediterranean diet in a sample of Portuguese adolescents. Perspect Public Health 131, 283-287.

39. Willett WC (2012) Nutritional Epidemiology, 3rd ed. New York: Oxford University Press.

40. National Institute of Diabetes and Digestive and Kidney Diseases (2016) Diabetes diet, eating, \& physical activity. https://www.niddk.nih.gov/health-information/diabetes/ overview/diet-eating-physical-activity (accessed July 2019).

41. World Health Organization (2000) Obesity: Preventing and Managing the Global Epidemic. Report of a WHO Consultation. WHO Technical Report Series no. 894. Geneva: WHO.

42. World Health Organization \& Food and Agriculture Organization of the United Nations (2004) Vitamin and Mineral Requirements in Human Nutrition. Report of a Joint FAO/WHO Expert Consultation, 2nd ed. Geneva: WHO.

43. Food and Agriculture Organization of the United Nations (2010) Fats and Fatty Acids in Human Nutrition. Report of an Expert Consultation. FAO Food and Nutrition Paper no. 91. Rome: FAO.

44. World Health Organization (2012) Guideline: Sodium Intake for Adults and Children. Geneva: WHO. 